\title{
Reconstruction of the superior vena cava with an autologous pericardial patch for a giant superior vena cava aneurysm
}

\author{
Kentaro Honda, MD, PhD, Mitsuru Yuzaki, MD, Takahiro Fujimoto, MD, and \\ Yoshiharu Nishimura, MD, PhD, Wakayama, Japan
}

\footnotetext{
From the Department of Thoracic and Cardiovascular Surgery, Wakayama Medical University, Wakayama, Japan. Disclosures: The authors reported no conflicts of interest.

The Journal policy requires editors and reviewers to disclose conflicts of interest and to decline handling or reviewing manuscripts for which they may have a conflict of interest. The editors and reviewers of this article have no conflicts of interest.

Received for publication Oct 14, 2020; accepted for publication Oct 14, 2020; available ahead of print Oct 16, 2020.

Address for reprints: Kentaro Honda, MD, PhD, Department of Thoracic and Cardiovascular Surgery, Wakayama Medical University, Kimiidera, Wakayama City, Wakayama, Japan (E-mail: honda-k@wakayama-med.ac.jp). JTCVS Techniques 2020;4:183-6

2666-2507

Copyright (c) 2020 The Authors. Published by Elsevier Inc. on behalf of The American Association for Thoracic Surgery. This is an open access article under the CC BY-NC-ND license (http://creativecommons.org/licenses/bync-nd/4.0/).

https://doi.org/10.1016/j.xjtc.2020.10.012
}

Video clip is available online.

A 54-year-old man was transported to our hospital as the result of a loss of consciousness. Emergent enhanced computed tomography (CT) revealed a large superior vena cava $(\mathrm{SVC})$ aneurysm $(62 \times 92 \times 72 \mathrm{~mm})$ and pulmonary thromboembolism. As the patient was hemodynamically stable, prudent follow-up with anticoagulant therapy was performed. During the follow-up, thromboembolism of the pulmonary artery improved; however, chest radiography performed 6 months after admission showed an enlarged SVC shadow (Figure 1, $A$ and $B$ ). CT revealed rapid expansion of the aneurysm $(65 \times 117 \times 88 \mathrm{~mm})$ into the right upper thoracic cavity, and surgery was planned. The orifice of the aneurysm (approximately $2 \mathrm{~cm}$ in size on enhanced CT) was located immediately above the junction of the azygos vein; patch repair with autologous pericardium was planned. Aortography was performed to exclude arteriovenous fistula, and no communication was detected.

Surgery was performed with the patient under general anesthesia. Under full sternotomy, the SVC aneurysm was confirmed between the pericardium and right-side parietal pleura (Figure 2, A). The wall of the aneurysm was extremely thin and was depressed and dilated on breathing. To enable safe and further dissection, cardiopulmonary

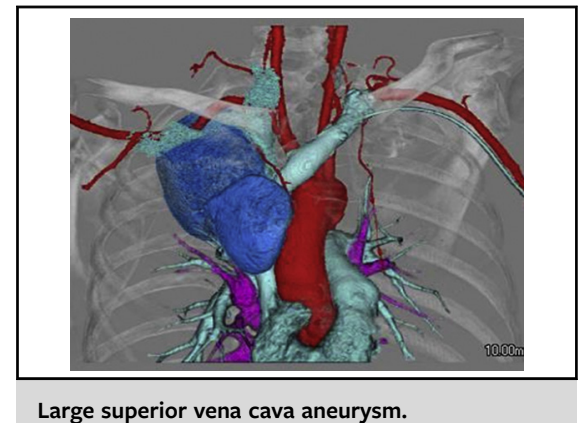

Large superior vena cava aneurysm.

\begin{abstract}
CENTRAL MESSAGE
Rupture and pulmonary thromboembolism are potentially lethal complications of a large superior vena cava aneurysm. Reconstruction of the venous wall with pericardium is a good treatment option.
\end{abstract}

See Commentary on page 187.

bypass was initiated to stop ventilation during dissection and reduce the risk of rupture. A venous drainage tube was inserted in the right and left brachiocephalic veins and inferior vena cava, and an arterial cannula was inserted in the ascending aorta. After the veins were clamped, the SVC was opened longitudinally (Figure 2, B). A thrombus was not observed inside the aneurysm cavity. The anterior, right, and posterior SVC wall, constituting $75 \%$ of the SVC, appeared damaged, thin, and dilated. The wall structure of the lesions differed from the structure of the remainder of the venous wall. Reconstruction of the SVC wall with an autologous pericardial patch was planned. The estimated dimeter and circumference of the SVC were 2 and $6.28 \mathrm{~cm}$, respectively, on preoperative CT. As $75 \%$ of the SVC was damaged, a $4-$ to $5-\mathrm{cm}$ wide patch was required. The pericardium was cut into $4-\times 6-\mathrm{cm}$ pieces for anterior, lateral, and posterior SVC wall reconstruction with 5-0 polypropylene continuous sutures (Figure 2,C). The use of xenogeneic tissue was avoided 


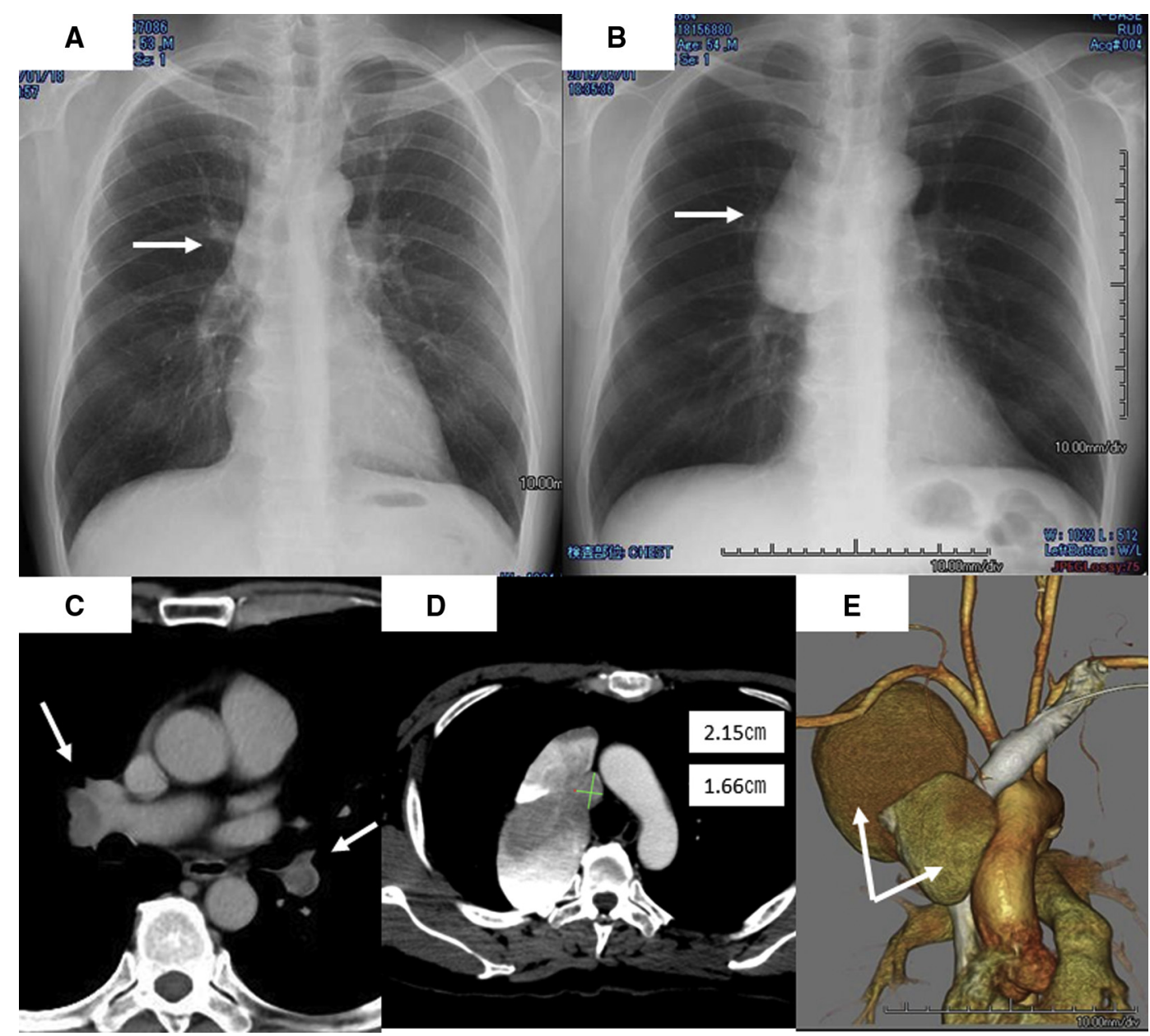

FIGURE 1. A and B, SVC aneurysm (white arrows) observed on chest radiographs recorded at admission and 6 months later. C, Thromboembolism of the right pulmonary artery. D and E, Multilocular large SVC aneurysm $(65 \times 117 \times 88 \mathrm{~mm})$. The estimated SVC diameter was approximately $2 \mathrm{~cm}$ on preoperative computed tomography.

in accordance with the patient's wish. Cardiopulmonary bypass weaning was uneventful, and the SVC pressure was normal (Video 1). No complications developed, and the postoperative course was uneventful. Pathologic examination of the aneurysm revealed normal venous wall structure with no localized wall structure abnormalities; however, the wall was thinner at the aneurysm site than at the other sites. CT at 6 months postoperatively revealed a well-reconstructed SVC (Figure 2, D). Informed consent for the publication of this paper was obtained from the patient.

\section{DISCUSSION}

SVC aneurysm is a rare venous pathology. Abbotto ${ }^{1}$ first described congenital aneurysm of the SVC in 1950 and with Leigh $^{2}$ reported the classification in 1963. Congenital etiologies, trauma, previous surgery, and other factors may lead to the development of an SVC aneurysm; however, this patient presented with no specific history, such as high-energy trauma of the chest wall, surgery, obstruction, neoplasms, and arteriovenous aneurysms.

The management of SVC aneurysms after diagnosis is also controversial. Some studies report that conservative treatment is beneficial, ${ }^{3}$ particularly for primary, small (maximum diameter of $<40 \mathrm{~mm}$ ), and fusiform aneurysms and asymptomatic cases. These studies indicate that the treatment strategy should be based on the type and size of the aneurysm, but identification of the aneurysm type is challenging. If conservative treatment is selected, CT follow-up is essential.

In this patient, SVC aneurysm was apparent on CT at admission but was difficult to identify on chest radiographs. Chest radiography is not suitable for prudent follow-up.

Another treatment option is endovascular therapy. Griviau and colleagues ${ }^{4}$ reported a case of successful endovascular therapy for SVC aneurysms. This less-invasive method is also a good treatment option for small aneurysms, but large aneurysms are more difficult to treat. 


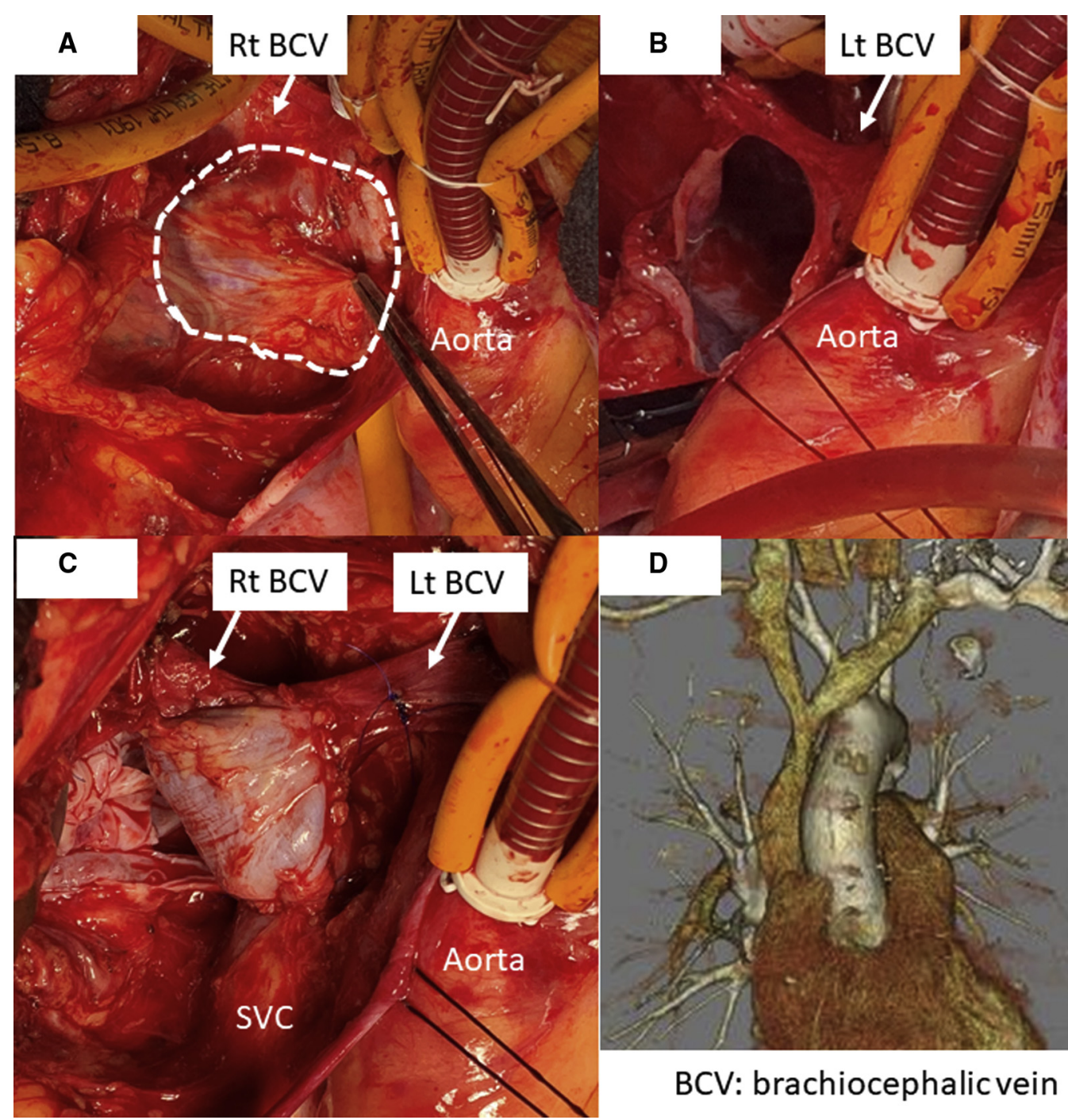

FIGURE 2. A, Intraoperative image of the SVC aneurysm. B, Deep aneurysmal cavity after longitudinal incision. C, Image obtained after SVC reconstruction with the pericardial patch. D, Computed tomography image of the reconstructed SVC at 1-year postoperatively. Rt, Right; $B C V$, brachiocephalic vein; $L t$, left; $S V C$, superior vena cava.

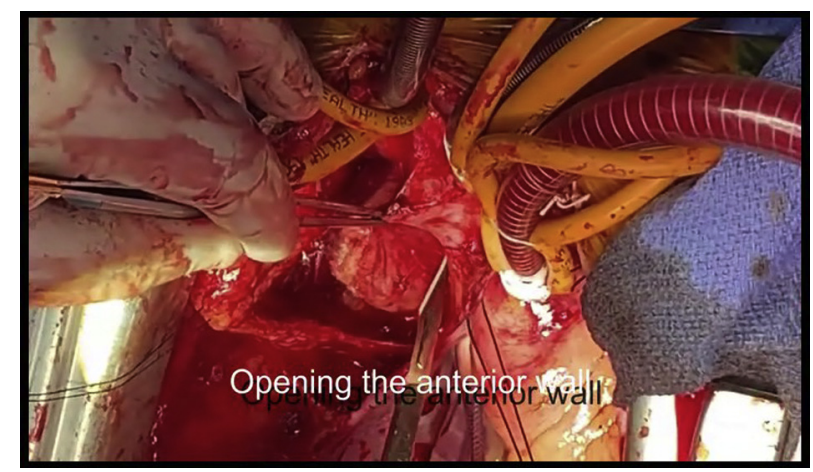

VIDEO 1. Large superior vena cava aneurysm and reconstruction of the superior vena cava with a fresh pericardial patch. Video available at: https://www.jtcvs.org/article/S2666-2507(20)30582-4/fulltext.
Another aspect of this disease is that a thrombus may form inside the aneurysm and cause pulmonary thromboembolism. In this patient with a large SVC aneurysm, bilateral pulmonary thromboembolism was detected. In patients with rapidly expanding SVC aneurysms, surgery may be the best treatment option, and with large aneurysms, the saphenous vein was too small to reconstruct the SVC. SVC reconstruction with an autologous pericardial patch is a good option for avoiding anticoagulant therapy. ${ }^{3,5}$

\section{References}

1. Abbotto OA. Congenital aneurysm of superior vena cava. Ann Surg. 1950;131: 259-63.

2. Abbotto OA, Leigh TF. Aneurysmal dilatations of the superior vena caval system Ann Surg. 1964;159:858-72. 
3. Janczak D, Skiba J, Gemel M, Mak M, Ziomek A, Malinowski M, et al. Giant saccular superior vena cava aneurysm - a rare and difficult clinical case. J Thorac Dis. 2016;8:E247-9.

4. Griviau L, Chevallier O, Favelier S, Pottecher P, Gehin S, Loffroy R Endovascular management of a large aneurysm of the superior vena cava involving internal thoracic vein with remodeling technique. Quant Imaging Med Surg. 2016;6:315-7.

5. Patel A, Cobb R, Rivera V, Simpson S. Untreated superior vena cava aneurysm: radiological significance and review of the literature. Case Rep Radiol. 2016; 2016:6960757. 